\title{
Harald Pors in Zusammenarbeit mit Ole Lauridsen, Bendt Falkes- gaard Pedersen und Flemming Talbo Stubkjær: Fortschritte. Di- daktisch-grammatisches Übungsbuch für den Hochschulunterricht. København: Aschehoug, 1998
}

Wer einmal das Angebot an Unterrichtsmaterial für den Grammatikunterricht an Hochschulen in Dänmark untersucht hat, wird wissen, daß es zwar eine Vielzahl von hausinternen Kompendien gibt, jedoch kaum ein eigentliches Angebot (Es wird hier von Übungsbüchern abgesehen, die für den gymnasialen Deutschunterricht geschrieben, jedoch im Hochschulunterricht eingesetzt werden, wie z.B. Lauridsen/Falkesgaard Pedersen/Pors 1995 [1992].). Ein didaktisch-grammatisches Übungsbuch für den Hochschulunterricht wie das vorliegende ist allein aus dem Grund mit großem Interesse und großer Freude willkommen zu heißen. Ich möchte auch schon eingangs feststellen, daß der Titel "Fortschritte" in mehreren Hinsichten zutrifft. So werden die Benutzer des Übungsbuches ohne Zweifel Fortschritte machen, aber auch das Übungsbuch an sich ist als ein Fortschritt zu sehen, zumal es alles in allem einen positiven Beitrag zum Grammatikunterricht an Hochschulen leistet.

Die Zielgruppe des Übungsbuches sind "Deutschlerner, die die elementare Grammatik in der Praxis beherrschen" (Vorwort), und die Übungen "bauen somit auf dem gymnasialen Niveau auf und konzentrieren sich auf Problembereiche und Fehlerquellen, die erfahrungsgemäß Studierenden auf der postgymnasialen Stufe im schriftlichen und mündlichen Gebrauch des Deutschen besondere Schwierigkeiten bereiten" (Vorwort). Obwohl das Übungsbuch auf Deutsch verfaßt ist, was - die Zielgruppe vor Augen gehalten - als positiv hervorzuheben ist, und deswegen grundsätzlich universell einsetzbar sein sollte, ist die eigentliche Zielgruppe des Übungsbuches eher dänischsprachige Deutschlerner auf der o.g. Stufe. Es wird nämlich viel Wert auf den kontrastiven Aspekt gelegt, zumal "die kontrastive sprachliche Situation des Fremdsprachenlerners einen wesentlichen Faktor des Erwerbsprozesses darstellt" (Vorwort), weshalb das Material auch "in zentralen sprachlichen Bereichen explizit oder implizit kontrastiv [i.e. Deutsch - Dänisch; MGD] angelegt [ist]" (Vorwort). Dies steuert zum Teil die Stoffselektion und lädt geradezu zum expliziten Vergleich mit Dänisch als Ausgangssprache ein, was als Stärke zu betrachten ist, zumal dadurch eine präzise und detaillierte Themenauswahl möglich ist. Es bedeutet jedoch umgekehrt nicht, daß das Übungsbuch nicht auch für andersprachige Deutschlerner von Interesse sein könnte.

Das übergeordnete Ziel des Übungsbuches - so läßt sich aus dem Vorwort schließen - ist es, das Korrektheitsniveau zu verbessern. Dabei ist es die Erfahrung der Verfasser, "daß das relativ hohe Korrektheitsniveau [...] sich am sichersten und effektivsten durch eine Bewußtmachung der relevanten sprach- 
lichen Strukturen erreichen läßt und daß die Einbeziehung der Abstraktionsund Systematisierungsfähigkeit des Lerners sinnvoll ist" (Vorwort). Vor diesem Hintergrund sind die Übungen zu sehen, die darauf abzielen, grammatische Strukturen bewußtzumachen und zu automatisieren, sprachliche Beobachtungen zu machen und grammatische Regeln daraus zu schließen und schließlich "die Lerner dazu anzuregen, kritische Benutzer von grammatischen Handbüchern und Nachschlagewerken und auch in sprachlicher Hinsicht aufmerksame Leser von deutschen Texten zu werden" (Vorwort). Zur Erreichung dieser Ziele werden unterschiedliche Übungsformen angeboten, wie Ergänzungs-, Umschreibungs-, Übersetzungsübungen sowie Übungen, die dem Kommentieren dienen. Auf die Übungsarten werde ich weiter unten zurückkommen. Den Übungen wird eine Bezugnahme auf relevante grammatische Handbücher vorausgeschickt. Es handelt sich hauptsächlich um die Grammatiken Rossen 1995 [1982], Jørgensen 1982 [1953-1964], Lauridsen/Poulsen 1995 sowie Duden 4. Wenn es aber im Vorwort heißt, daß "auf grammatische Handbücher verwiesen [wird], die im dänischen postgymnasialen Deutschunterricht Anwendung finden", müßte wohl eigentlich auch auf Collin Eriksen/ Bruun Hansen/Lantow/Stenestad 1989 [1984] verwiesen werden. Es geht im übrigen nicht hervor, nach welchen Kriterien die einzelnen Grammatiken eingesetzt werden, was aber in der Praxis keineswegs problematisch erscheint. Es sieht jedoch so aus, als sei Rossen 1995 [1982] die Basisgrammatik, und als seien die übrigen zur Unterstützung bzw. Ergänzung herangezogen. Als Stärke des Übungsbuches ist zu bewerten, daß an Stellen, wo die Grammatiken sozusagen versagen, explizite Regeln angeführt werden. Als Beispiel sei hier Übung 181 genannt, in der die Tempuswahl in der indirekten Rede näher erläutert wird.

Das verwendete Material sind "weitgehend deutsche und dänische Originalbelege oder adaptierte Originalbelege aus unterschiedlichen Textkorpora" (Vorwort), was als äußerst positiv zu sehen ist, weil im weiten Umfang konstruierte Beispiele dadurch vermieden werden können. In dem Zusammenhang möchte ich noch als positiv hervorheben, daß die Verfasser auch, wo möglich, nicht nur isolierte Einzelsätze, sondern zusammenhängende Textstücke eingesetzt haben, so z.B. in Übung 18 zum Genus der Substantive oder in Übung 69 zur Übersetzung von “det er - der er”. Es leuchtet ein, daß es wegen des Detaillierungsgrads der Themenauswahl kaum möglich ist, für die spezifischten Themen wie z.B. die deutschen Äquivalente von “bygge på” (Übung 151) Textstücke zu finden, die als Übungstexte fungieren können. Was das Material betrifft, läßt sich übergeordnet sagen, daß es durchaus den deutschen bzw. dänischen Sprachgebrauch im allgemeinen wiederspiegelt, nur erscheint es mir ein wenig problematisch, ihn - wie es im Vorwort getan wird - wegen der Einbeziehung von Originalbelegen aktuell zu benennen, wenn man bedenkt, 
daß drei der insgesamt sechs verwendeten Korpora aus den Jahren vor 1975 stammen.

Das Übungsbuch gliedert sich in fünf übergeordnete Kapitel. Im Kapitel Morphologie sind Übungen zu beispielsweise Pluralbildung und Genus der Substantive, Deklination und Komparation der Adjektive, Flexionsformen der Verben und schließlich zu Flexion der Pronomina zu finden. Hier finden sich auch Übungen, die den kontrastiven Aspekt besonders deutlich machen, und zwar solche, die die Problematik dänischer Pronomina wie "den, der" oder "hinanden” und ihrer deutschen Äquivalente aufgreifen. Solche explizit kontrastiv angelegten Übungen gibt es in sämtlichen Kapiteln außer im Kapitel über den Konjunktiv (s.u.), weswegen in der weiteren Präsentation der einzelnen Kapitel nur dann darauf eingegangen wird, wenn die kontrastiven Übungen von besonderem Interesse sind.

Im Kapitel Kasus geht es um die Funktionen der Kasus Nominativ, Akkusativ, Dativ und Genitiv sowie den Kasusgebrauch bei Appositionen.

Im Kapitel Präpositionen: Gebrauch und Rektion gibt es - wie der Titel schon besagt - Übungen nicht nur zur Rektion, wie es sonst hauptsächlich der Fall ist, sondern auch Übungen zum Gebrauch der Präpositionen. Es geht dabei primär um die Wahl der Präposition nach bzw. vor Verben, Substantiven und Adjektiven, und somit lassen sich diese Übungen auch unter dem Aspekt der Valenz betrachten. Mir gefällt es sehr, daß Übungen zur Wahl der Präpositionen Platz gefunden haben, zumal eben diese Thematik bekanntlich zu den schwierigsten Bereichen des Fremdsprachenerwerbs gehört. Überhaupt ist das Kapitel über die Präposition beispielhaft für das ganze Übungsbuch, indem hier die Relevanz, die Innovation, die Spezifiziertheit sowie die Kontrastivität deutlich werden. Außer den Übungen zur Wahl der Präposition, die relevant und als Übungstyp auch innovativ sind, sind die kontrastiv angelegte Übung zur Übersetzung von "før (inden)”, die Übung zum Kasus im Zusammenhang mit "der alten Bewegung" sowie die Übung zum Präpositionalgefüge als Attribut, hervorzuheben, weil diese sowohl relevant, innovativ, spezifisch und zum Teil auch kontrastiv sind. Darüber hinaus enthält das Kapitel Übungen zum Thema Schwankungen in der Rektion, die aus dem Grunde erwähnenswert sind, weil sie besonders deutlich machen, daß es sich um ein Übungsbuch für Studenten auf dem postgymnasialen Niveau handelt. Das Thema an sich gibt an, daß es sich nicht länger um die Basisregeln handelt, sondern um einen komplexeren Bereich ohne eindeutige Antworten. Hier werden Korpusbelege auch dadurch bestens genutzt, daß eine Übung wie die folgende zu finden ist: "Untersuchen Sie in folgenden Korpusbelegen, ob bei aufbauen auf usw. der häufigere oder der weniger häufige Kasus [gemäß der vorangehenden Darstellung; MGD] verwendet ist" (S.149). 
Das Kapitel Konjunktiv bietet Übungen zur Form und Funktion des Konjunktivs I und II an, und zwar Übungen zur großen Palette der Einsatzmöglichkeiten des Konjunktivs. Wie schon erwähnt, gibt es keine explizit kontrastiv angelegten Übungen in diesem Kapitel, wobei aber gesagt werden muß, daß die einzelnen Problemstellungen der dänischsprachigen Deutschlerner im Bereich des Konjunktivs wohl auch weniger aus kontrastiver Sicht zu lösen sind. Wie im Rest des Übungsbuches varriiert auch in diesem Kapitel die Länge der Übungen. Es wirkt befreiend, daß es keine magische Anzahl von Beispielen gibt. So gibt es z.B. Übungen mit nur vier (Übung 159) bzw. neun Beispielsätzen (Übung 166), solche mit 17 (Übung 173) oder 41 (Übung 192), aber auch wiederum Übungen ohne Nummerierung (vor allem Übungen, die auf ganzen Textstücken basiert sind, wie Übung 184 - 186). Die Länge der Übungen ist zu Recht offensichtlich vom Detaillierungsgrad, Übungstyp und der Anzahl von Korpusbelegen abhängig. Man kann sich jedoch die Frage stellen, wann eine Übung eine Übung ist. So kommen Übungen vor, die aus mehreren Teilübungen bestehen, wie Übung 170, und solche, die keine Übung im eigentlichen Sinne enthalten wie Übung 181, in der allein informiert wird. Diese Problematik spielt in der Praxis jedoch kaum eine Rolle.

Im letzten Kapitel Wortstellung wird zunächst ein vereinfachtes Wortstellungsschema introduziert, daraufhin wird die Wortstellung innerhalb der einzelnen Wortstellungsfelder (Vor-, Schluß-, Mittel und Nachfeld) behandelt. Darüber hinaus gibt es ein paar Übungen zur Wortstellung innerhalb von Satzgliedern, ein sonst eher vernachlässigtes Gebiet, sowie die in kontrastivem Licht unvermeidbaren Übungen zur Satzspaltung, Schachtelung, Satzverschränkung (Satzknoten) sowie zur Wortstellung innerhalb von Infinitivkonstruktionen.

Das Übungsbuch bietet verständlicherweise keine Progression der übergeordneten Themen an, so wird zu Recht - die Zielgruppe vor Augen gehalten z.B. die Adjektivdeklination als bekannt vorausgesetzt in Übung $7 \mathrm{im} \mathrm{Ab-}$ schnitt über die Deklination der Substantive, obwohl die Adjektivdeklination erst im nachfolgenden Abschnitt thematisiert wird. Dies bedeutet, daß die einzelnen Themen je nach Bedarf ausgewählt werden können, was eine weitere Stärke des Übungsbuches ist. Wie gezeigt enthält das Übungsbuch Übungen zu den unterschiedlichsten nicht zur Elementargrammatik gehörenden Themen zwecks Bewußtmachung und Automatisierung morphologischer und syntaktischer Strukturen (vgl. oben sowie Vorwort). Es bietet sich daher die Frage, die eine viel eingehendere Diskussion verdient, als hier zu führen ist, und zwar inwieweit die angebotenen Übungen generell gesehen ausreichen, um die obenerwähnten Regularitäten nicht nur bewußtzumachen, sondern auch zu automatisieren. Nehmen wir ein Beispiel wie die Ergänzungsübung 144 zur Kasuswahl nach "in" bei Verben wie "ankommen in", die aus elf Beispielsät- 
zen besteht. Ich bezweifle keineswegs, daß die Problematik bewußtgemacht wird, sondern allein inwieweit elf Beispielsätze ausreichen, um die Automatisierung syntaktischer Regularitäten zu gewährleisten. Die zu beantwortenden Fragen wären dann, wieviele Beispielsätze einer solchen Automatisierung zugrundeliegen und inwieweit sich die Automatisierung nur durch wiederholte Übungen erzielen läßt?

Der obengenannte Übungstyp Ergänzung läßt sich zusammen mit den Übungstypen Übersetzung und Umschreibung als sogenannte produktive Übung charakterisieren. In Ambjørn 1998 wird zwischen diesen sogenannten produktiven Übungen, die produkt- oder auch outputorientiert sind, und sogenannten rezeptiven oder auch verständnisorientierten Übungen, die umgekehrt prozeß- oder auch input-/intakeorientiert sind, unterschieden. Unter einer rezeptiven Übung versteht Ambjørn eine Übung, die das Verständnis des Fremdsprachenerwerbers bezweckt und die sich von einer produktiven dadurch unterscheidet, daß sie auf keine Form der Fremdsprachenproduktion abzielt (Ambjørn 1998: 52). Um das Ziel der rezeptiven Übungen zu verdeutlichen, ist es wichtig zuerst die beiden Begriffe Input und Intake zu erläutern. Der Input wird von fremdsprachlichen Daten der mündlichen und schriftlichen Kommunikation sowie durch Bewußtmachung sprachlicher Strukturen geliefert (Ambjørn 1998: 50). Das Intake dagegen ist allein das, was die Fremdsprachenerwerber vom Input verstehen, jedoch nicht notwendigerweise in der Praxis beherrschen (Ambjørn 1998: 50). Das Ziel der rezeptiven Übungen ist es nun, die Inputverarbeitung zu optimieren und dadurch das Intake zu fördern (Ambjørn 1998: 51). Als rezeptive Übungsformen schlägt Ambjørn (1998: 52) konkret folgende Übungsformen vor, die alle im hausinternen Kompendium für den rezeptiven Teil des Grammatikunterrichts auf dem BAStudium am Institut für Spanisch, Wirtschaftsuniversität Aarhus, zu finden sind (=Ambjørn/Andersen 1998): Beantwortung von Fragen, Identifikation von Phänomenen, Erkennung von Zusammenhängen zwischen Form und Bedeutung / Funktion, Ableitung von Regeln, Beschreibung von Phänomenen, Erklärung von Phänomenen aus kontrastiver Sicht und schließlich Diskussion und Lösung von Problemstellungen. Auch das vorliegende Übungsbuch zeichnet sich dadurch aus, daß es eine große Anzahl von solchen rezeptiven Übungen gibt. So heißt es beispielsweise "Beschreiben Sie die syntaktische Funktion der kursiv gedruckten Adjektive in folgenden Beispielen [...]" (Übung 33) oder "Untersuchen Sie den Kasusgebrauch bei kosten mit zwei Objekten in den Beispielen 1-41, und überlegen Sie, ob der Kasusgebrauch mit der Regel übereinstimmt, die Duden 9 anführt? Überlegen Sie auch, warum Belege wie a-c in diesem Zusammenhang nicht brauchbar sind?" (Übung 82). Das Vorkommen von rezeptiven Übungen wird aus dem Grunde als positiv betrachtet, weil dadurch eine Verständnisphase einbezogen wird nach einer 
Bewußtmachungsphase und vor einer Automatisierungsphase als den beiden Phasen, die traditionell berücksichtigt werden. Die Einbeziehung einer Verständnisphase verbessert nämlich den Output, wie sich aufgrund psycholinguistischer Analysen im Bereich des Fremdsprachenerwerbs schließen läßt (gemäß Ambjørn 1998, wo Hinweise auf Literatur zum Thema zu finden sind). Diese Einsicht müßte meines Erachtens auf Sicht für zukünftige Materialien für den Grammatikunterricht Konsequenzen haben. So sollte schon in der Konzeptionsphase entschieden werden, inwieweit das zu entwickelnde Unterrichtsmaterial zur Unterstützung sämtlicher oder ausgewählter Phasen beitragen soll. Diese Entscheidung wird dann logischerweise einen Einfluß auf die Themenbreite haben, zumal die Berücksichtigung sämtlicher Phasen weit mehr Übungen fordert als die Berücksichtigung einer Phase. Für das vorliegende Übungsbuch bleibt in dem Zusammenhang nur festzuhalten, daß sowohl rezeptive als auch produktive Übungen vorkommen, daß jedoch nicht für jedes Thema beide Arten angeboten werden (was bei der dargebotenen Themenvielfalt auch nicht zu erwarten wäre) und schließlich, daß die produktiven Übungen nicht immer den rezeptiven folgen.

Zusammenfassend ist festzustellen, daß das Übungsbuch für den Grammatikunterricht auf der postgymnasialen Stufe empfehlenswert ist. So bietet es, was die Themen betrifft, auf der einen Seite eine breite Auswahl, auf der anderen Seite Übungen zu spezifischen Problemstellungen, die für die Zielgruppe äußerst relevant sind. Darüber hinaus bietet es eine variierte Auswahl an Übungsformen, von denen ich besonders die Übungen zur Bewußtmachung und Beobachtung sprachlicher Konstruktionen hervorheben möchte. Das Übungsbuch ist traditionell im Kontaktunterricht einsetzbar. Dazu kommt, daß große Teile des Übungsbuches dazu einladen, es auch für das Selbststudium zu verwenden - worauf die Verfasser auch selbst im Vorwort aufmerksam machen - und dies vor allem, nachdem ein Lösungsschlüssel vorliegt.

\section{Literatur}

Ambjørn, Lone (1998). Reception før produktion - proces før produkt. In Sprogforum. Tidsskrift for sprog- og kulturpaedagogik 10. 50-55.

Ambjørn, Lone \& Andersen, Vibeke (1998). Receptive фvelser til grammatikundervisningen $i$ spansk (=Internt undervisningsmateriale $n r . R$ 138). Spansk Institut, Handelshøjskolen i Århus.

Collin Eriksen, C., Bruun Hansen, Agnete, Lantow, Bent \& Stenestad, Elva (1989 [1984]). Gyldendal Tysk Grammatik. København: Nordisk Forlag A.S.

Duden 4 (1995). Duden. Grammatik der deutschen Gegenwartssprache (=Duden Band 4). Herausgegeben und bearbeitet von Günther Drosdowski in Zusammenarbeit mit Peter Eisenberg, Hermann Gelhaus, Helmut Henne, Horst Sitta und Hans Wellmann. Mannheim usw.: Dudenverlag. 
Duden 9 (1985). Duden. Richtiges und gutes Deutsch (=Duden Band 9). Bearbeitet von Dieter Berger und Günther Drosdowski unter Mitwirkung von Otmar Käge und weiteren Mitarbeitern der Dudenredaktion. Mannheim usw.: Dudenverlag.

Jørgensen, Peter (1992 [1953-1964]). Tysk Grammatik I - III. København: G.E.C. Gads Forlag.

Lauridsen, Ole, Falkesgaard Pedersen \& Pors, Harald (1995 [1992]). In der Praxis. Arbejdsbog til skriftlig og mundtlig tysk. København: Aschehoug Dansk Forlag A/S.

Lauridsen, Ole \& Poulsen, Sven-Olaf (1995). Tysk Grammatik. København: Munksgaard.

Rossen, A. (1995 [1982]). Tysk Grammatik. Oversat og bearbejdet af P.V. Christiansen, Bendt Pedersen og Harald Pors. København: Aschehoug.

Marianne Grove Ditlevsen 